\title{
Signature of pseudodiffusive transport in mesoscopic topological insulators
}

\author{
Saurav Islam $\odot,{ }^{1, *}$ Semonti Bhattacharyya $\odot,{ }^{1, \dagger}, *$ Hariharan Nhalil, ${ }^{1}$ Suja Elizabeth, ${ }^{1}$ and Arindam Ghosh ${ }^{1,2}$ \\ ${ }^{1}$ Department of Physics, Indian Institute of Science, Bangalore 560012, India \\ ${ }^{2}$ Centre for Nanoscience and Engineering, Indian Institute of Science, Bangalore 560012, India
}

(Received 11 January 2020; accepted 3 June 2020; published 6 July 2020)

\begin{abstract}
One of the unique features of Dirac Fermions is pseudodiffusive transport by evanescent modes at low Fermi energies when disorder is low. At higher Fermi energies, i.e., charge carrier densities, the electrical transport is diffusive in nature and the propagation occurs via plane waves. In this study, we report the detection of such evanescent modes in the surface states of topological insulator through $1 / f$ noise for the first time. While signatures of pseudodiffusive transport have been seen experimentally in graphene, such behavior is yet to be observed explicitly in any other system with a Dirac dispersion. To probe this, we have studied $1 / f$ noise in topological insulators as a function of gate voltage, and temperature. Our results show a nonmonotonic behavior in $1 / f$ noise as the gate voltage is varied, suggesting a crossover from pseudodiffusive to diffusive transport regime in mesoscopic topological insulators. The temperature dependence of noise points towards conductance fluctuations from quantum interference as the dominant source of the noise in these samples.
\end{abstract}

DOI: 10.1103/PhysRevResearch.2.033019

\section{INTRODUCTION}

Topological insulators (TIs), with their spin-polarized, topologically protected, linear, metallic surface states, act as the perfect playground for investigating a plethora of fundamental phenomena [1-5]. These surface carriers obey the Dirac equation for massless Fermions, where the Hamiltonian of the system is given by $H=\hbar v_{F} \vec{\sigma} \cdot \vec{k}$. Here $v_{F}, \vec{\sigma}$, and $\vec{k}$ refer to the Fermi velocity, spin matrices, and momentum, respectively. Due to the massless nature of the charge carriers, the screening properties of Dirac materials such as TIs or graphene, are also significantly different from traditional twodimensional electron systems, and the potential due to charged disorder remains long ranged even after screening is taken into account [6]. Another key feature of these materials is the possibility of reaching $\langle n\rangle=0$, without opening up a band gap, even though strong carrier inhomogenity in the form of electron-hole puddles might be present around charge neutrality point or the Dirac point [7]. The electrical transport properties of these classes of materials near the Dirac point, where the Fermi surface diminishes to a point, has been a matter of intense discourse, and has led to several fascinating discoveries in the context of graphene, such as dissipative

\footnotetext{
*isaurav@iisc.ac.in

${ }^{\dagger}$ Present address: School of Physics and Astronomy, Monash University, Victoria 3800, Australia.

Present address: ARC Centre of Excellence in Future Low-Energy Electronics Technologies (FLEET), Monash University, Monash, Victoria 3800, Australia.

Published by the American Physical Society under the terms of the Creative Commons Attribution 4.0 International license. Further distribution of this work must maintain attribution to the author(s) and the published article's title, journal citation, and DOI.
}

quantum Hall effect, minimum conductivity, and pseudodiffusive transport [8-30].

Accessing the Dirac point in TIs compared to graphene has been a challenge due to high doping from bulk defects as well as the substrate, thus making it difficult to probe the intriguing properties of Dirac Fermions in TIs including the origin of $1 / f$ noise, which is generally attributed to the slow reorganization of background potential landscape [31-34]. The relaxation process is a superposition of fluctuators of characteristic time $\tau$ with a distribution $D(\tau) \propto \tau^{-1}$, and the magnitude of noise is given by the Fourier transform of the autocorrelation function of the fluctuating signal, defined as the power spectral density (PSD). Previous investigation of $1 / f$ noise in TIs have revealed the role of bulk disorder-mediated Hooge type mobility fluctuation type noise in 100-nm-thick mesoscopic samples, and correlated mobility-number density fluctuation model to be the dominant mechanism in large-area epitaxially grown samples [8,35-41]. However, the origin of $1 / f$ noise in TIs in thin (thickness $d \sim 10 \mathrm{~m}$ ) mesoscopic samples, especially near the Dirac point, remains a matter of debate. In this manuscript, we have explored the origin of $1 / f$ noise in mesoscopic samples, where we have access to the Dirac point. Our investigation has revealed sample-dependent nonmonotonic, as well as monotonic dependence of $1 / f$ noise magnitude on the charge carrier density, suggesting a crossover from pseudodiffusive to diffusive transport, and the conductance fluctuations from quantum interference effects as the main source of noise in these types devices at low $T$.

\section{EXPERIMENTAL DETAILS}

The topological insulator field-effect transistors (TI-FET) studied in this manuscript were fabricated using the TI $\mathrm{Bi}_{1.6} \mathrm{Sb}_{0.4} \mathrm{Te}_{2} \mathrm{Se}$ (BSTS), which is a compensation doped quarternary alloy, with an insulating bulk resulting in enhanced surface transport $[35,42,43]$, and below temperature $T<50 \mathrm{~K}$ for samples with thickness, $d \leqslant 100 \mathrm{~nm}$, the 


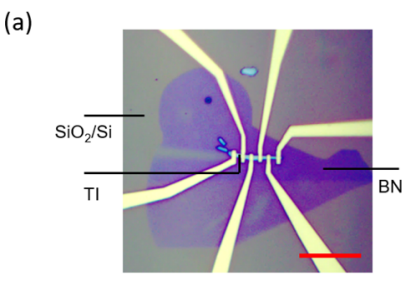

(b)
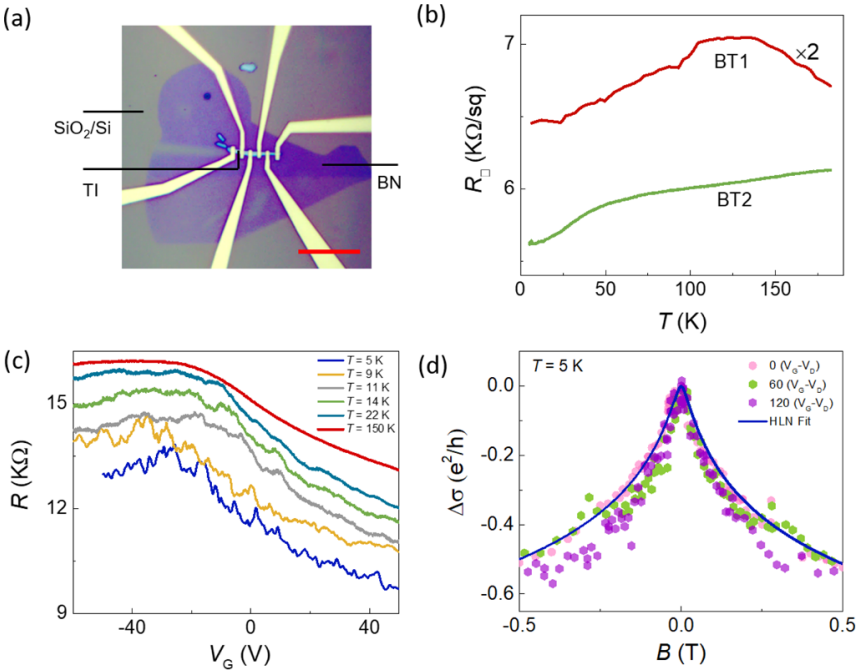

FIG. 1. Basic device characteristics. (a) Optical micrograph of a typical topological insulator field-effect transistor (TI-FET), transferred onto a boron nitride substrate. The scale bar is $5 \mu \mathrm{m}$. (b) Sheet resistance $\left(R_{\square}\right)$ vs temperature $(T)$ of both samples BT1, and BT2, displaying metallic behavior as $T$ is lowered. The data for BT1 has been multiplied by a factor of two. (c) Resistance $(R)$ vs gate voltage $\left(V_{G}\right)$ of sample BT1, at different $T$, showing ambipolar transport. The $R-V_{G}$ data for $T=9,11,14,22$, and, $150 \mathrm{~K}$ data has been shifted vertically by $1,1.5,2$, and $2.2 \mathrm{~K} \Omega$ respectively for clarity. (d) Weak-antilocalization in BT1, characterized by a cusp in the quantum correction to conductivity at $B=0 \mathrm{~T}$. The solid blue line is the fit to the data for $V_{G}-V_{D}=0 \mathrm{~V}$, using Eq (1).

current is essentially carried by the surface carriers [35]. The material was exfoliated from a single crystal using Scotch tape technique, and was transferred using a homemade heterostructure fabrication assembly, onto an atomically flat boron nitride $(\mathrm{BN})$ substrate, which significantly reduces the effect of dangling bonds and charged traps of the $\mathrm{SiO}_{2}$ substrate on the electrical transport in the TI channel [38,44-46]. The heterostructure was then finally assembled onto a prepatterned heavily doped $\mathrm{SiO}_{2} / \mathrm{Si}$ substrate, with the 285- nm- thick $\mathrm{SiO}_{2}$ acting as a back-gate dielectric. The sample contacts were patterned by standard electron-beam lithography, followed by thermal evaporation of 5/40 $\mathrm{nm} \mathrm{Cr} / \mathrm{Au}$. A layer of the polymer PMMA [poly(methylmethacrylate)] was coated on the samples, which ensured that the surface integrity is preserved throughout the measurement cycle. The optical micrograph of a typical TI-FET is shown in Fig. 1(a). The measurements reported in this manuscript were performed on two identically prepared samples, BT1 and BT2, in a homebuilt variable temperature cryostat. The resistivity measurements were performed using a low frequency AC-four probe technique with carrier frequency of $18 \mathrm{~Hz}$ with an excitation current of $100 \mathrm{nA}$.

\section{A. Preliminary electrical characteristics}

The sheet resistivity $\left(R_{\square}\right)$ vs temperature $(T)$ for both samples BT1 and BT2, as shown in Fig. 1(b), are quantitatively as well as qualitatively similar, displaying metallic behavior with reducing $T$, as expected for $\sim 10 \mathrm{~nm}$ thin TIs flakes [Fig. 1(b)] [35]. The difference in the sheet resistivity can be due to the difference in impurity density in the samples. The resistance vs gate voltage $\left(V_{G}\right)$ for sample BT1 is shown in Fig. 1(c), where a maxima in $R$ at $V_{G} \approx-40 \mathrm{~V}$ at $T=5 \mathrm{~K}$ represents the Dirac point. The asymmetry in the $R-V_{G}$ on the electron and holes sides may arise due to asymmetry in the band-structure itself [47]. The typical field-effect mobility extracted from the $R-V_{G}$ graph is $\sim 100 \mathrm{~cm}^{2} \mathrm{~V}^{-1} \mathrm{~s}^{-1}$. Figure 1(d) shows the magnetoresistance (MR) behavior of BT1 at $V_{G}-V_{D}=0,60$, and $120 \mathrm{~V}$, characterized by a cusp in the quantum correction to conductivity $\triangle \sigma$ at $B=0 \mathrm{~T}$ [46,48-51], due to weak-antilocalization phenomenon, as expected for spin-momentum locked TI surface states, resulting from an additional $\pi$ Berry phase between the back-scattered, time reversed path of the charge carriers leading to negative magnetoconductance. The magnetoconductance data can be fitted with the Hikami-Larkin-Nagaoka $[52,53]$ equation for diffusive metals with high spin orbit coupling $\left(\tau_{\phi} \gg \tau_{\text {so }}, \tau_{e}\right)$

$$
\triangle \sigma=-\alpha \frac{e^{2}}{\pi h}\left[\psi\left(\frac{1}{2}+\frac{B_{\varphi}}{B}\right)-\ln \left(\frac{B_{\varphi}}{B}\right)\right],
$$

where $\tau_{\phi}, \tau_{\mathrm{so}}, \tau_{e}$ are the phase coherence or dephasing time, spin-orbit scattering time and elastic scattering time, respectively, $\psi$ is the digamma function and $B_{\phi}$ is the phase breaking field. Here $\alpha$ and $B_{\phi}$ are fitting parameters. The phase coherence length $l_{\phi}^{\mathrm{MR}}$ can be extracted using $l_{\phi}^{\mathrm{MR}}=\sqrt{\hbar / 4 e B_{\phi}}$. The $l_{\phi}$ obtained from the fit was $\sim 180 \mathrm{~nm}$ at $T=5 \mathrm{~K}$ for $V_{G}-V_{D}=0 \mathrm{~V}$.

\section{B. Signature of pseudodiffusive transport}

In order to understand the origin of $1 / f$ noise in mesoscopic TIs, and extract the magnitude accurately, we have utilized the AC four-probe technique [32,33,54], as shown in Fig. 2(a). The experimental process involves recording the voltage fluctuations in a constant current circuit, as a function of time from the output of the lockin amplifier (SR 830) $[34,36,37,55]$. This is followed by digital signal processing, and decimation of the time-series data to obtain the PSD $\left(S_{V}\right)$ as a function of frequency $(f)$, using Welch's method of periodogram [56]. In both the devices BT1 and BT2, $S_{V} \propto$ $1 / f^{\alpha}$, and the exponent of the frequency, $\alpha \approx 1$ [Fig. 2(b)]. Additionally, $S_{V}$ depends on the bias across the sample $(V)$ in a quadratic manner, which ensured that all the measurements were performed in the Ohmic regime [Fig. 2(c)].

The $V_{G}$ dependence of the integrated noise magnitude $\left(\frac{\left\langle\delta G^{2}\right\rangle}{G^{2}}=\int \frac{S_{v}}{V^{2}} d f\right)$ at $T=5 \mathrm{~K}$, is shown in Figs. 3(a) and 3(b) for samples BT1 and BT2, respectively. Although these two samples were identically prepared from the same bulk crystal, and show similar average electrical characteristics, they demonstrate contrasting behaviors in the $V_{G}$ dependence of noise. Whereas $\frac{\left\langle\delta G^{2}\right\rangle}{G^{2}}$ vs $V_{G}$ for sample BT1 displays a nononotonic behavior with a peak around the Dirac point (| $V_{G}-V_{D} \mid=20 \mathrm{~V}$ ) [Fig. 3(a)], the identically prepared device BT2 shows a monotonic reduction as $V_{G}$ is tuned away from the Dirac point, as demonstrated in Fig. 3(b). The nonmonotonic behavior of $1 / f$ noise previously reported in the context of graphene [8] and in TIs [35,37], has been attributed to the interplay of charge exchange noise (originating due to exchange of carriers between the channel and the surrounding environment) and configuration noise (arising due to potential 

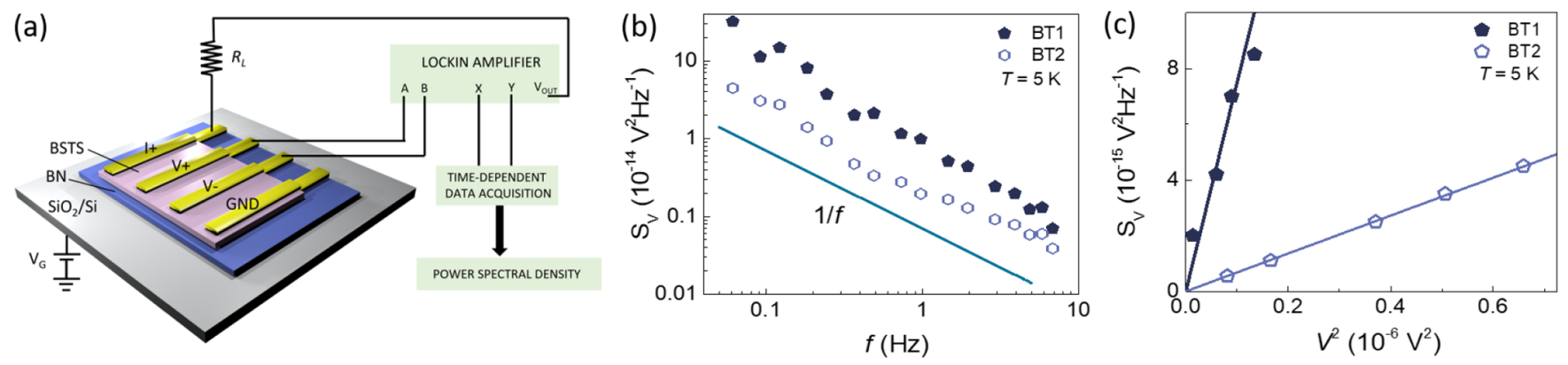

FIG. 2. Basic characteristics of $1 / f$ noise measurements. (a) Schematic of the experimental setup for measuring $1 / f$ noise. The voltage fluctuations are recorded in a constant current circuit, where $R_{L} \gg R_{\text {sample }}$ is used as the current limiting resistance. (b) Power spectral density $\left(\mathrm{S}_{V}\right)$ as a function of frequency, showing $1 / f$ behavior. (c) $\mathrm{S}_{V}$ vs $V^{2}$ at $T=5 \mathrm{~K}$ for both samples BT1 and BT2, showing a quadratic behavior, implying that the response is in the linear regime. Here $V$ is the bias across the sample.

fluctuations due to reorganization of trapped charges). We have fitted the $V_{G}$ dependence of the normalized noise magnitude data using the framework of correlated mobility-charge carrier density fluctuations model [37,57], which is known to be the dominant mechanism of noise in large-area, thin $(\sim 10 \mathrm{~nm})$ TIs, where the effect of conductance fluctuations are suppressed due to a large $L / l_{\phi}$ ratio. The total noise can be expressed as

$$
\frac{S_{V}}{V^{2}}=\frac{D_{i t} k_{B} T}{d W L}\left(\frac{d \sigma}{d n}\right)^{2}\left(\frac{J_{1}}{\sigma^{2}}+\frac{J_{2}}{\sigma}+J_{3}\right)
$$

where $J_{1}=\frac{1}{8 \alpha}$ represents a pure number fluctuation, $J_{3}=$ $\int A^{2}(x) \frac{\tau_{T}^{8 \alpha}}{1+\left(2 \pi f \tau_{T}\right)^{2}} d x$ represents pure mobility fluctuations, and $J_{2}=\int 2 A(x) \frac{\tau_{T}}{1+\left(2 \pi f \tau_{T}\right)^{2}} d x$ represents combined number and mobility fluctuations ( $\alpha$ is the decay constant for the spatially decaying time constant $\tau_{T}$ of a typical trapping event and $A(x)$ is the scattering constant) and can be evaluated using phenomenological values [57]. $D_{i t}, k_{B}, W, L, \sigma, n, x$, and $d$ are the areal trapped charge density per unit energy, Boltzmann constant, width of the channel, length of the channel, conductance, charge carrier density, axis in the direction perpendicular to the channel, and $d=1 \mathrm{~nm}$ is the distance over which the tunneling is effective. As evident from the fit, this framework does not satisfactorily explain the observed nature of $1 / f$ noise, implying that the dominant source of voltage fluctuations in mesoscopic samples and
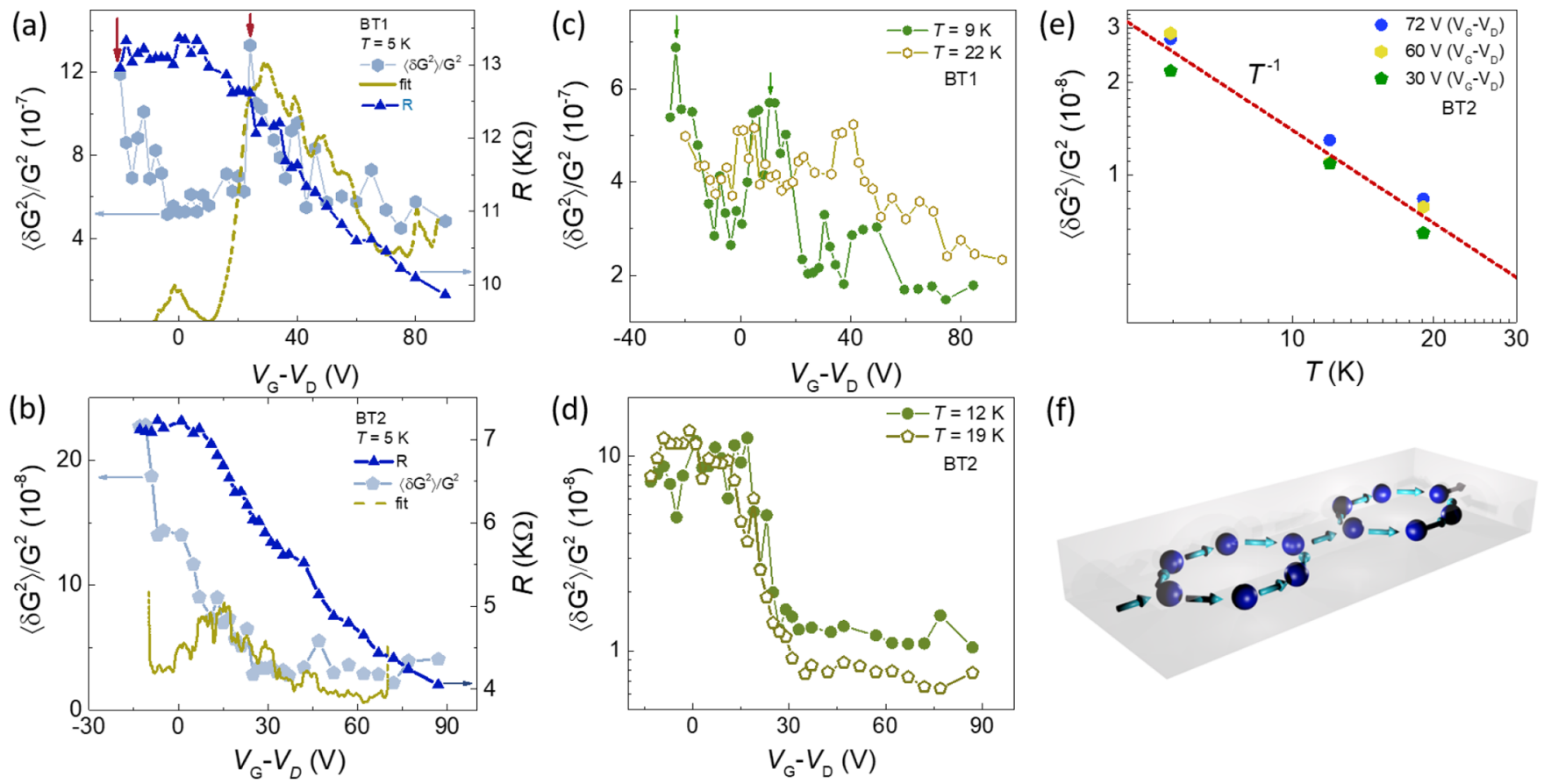

FIG. 3. $1 / f$ noise measurements. (a) Integrated noise magnitude $\frac{\left\langle\delta G^{2}\right\rangle}{G^{2}}$ as a function of gate voltage $\left(V_{G}\right)$ for sample BT1. The dashed line shows the fit according to Eq. (2). (b) $\frac{\left\langle\delta G^{2}\right\rangle}{G^{2}}$ as a function of $V_{G}$ for sample BT2. The dashed dark-yellow line shows fit according to Eq. (2). (c) $V_{G}$ dependence of $\frac{\left\langle\delta G^{2}\right\rangle}{G^{2}}$ at $T=9$ and $22 \mathrm{~K}$ for sample BT1. (d) $\frac{\left\langle\delta G^{2}\right\rangle}{G^{2}}$ as a function of $V_{G}$ for $T=12$ and $19 \mathrm{~K}$ for sample BT2. (e) $\frac{\left\langle\delta G^{2}\right\rangle}{G^{2}}$ vs $T$ for BT2, at $V_{G}-V_{D}=72 \mathrm{~V}, 60 \mathrm{~V}$, and $30 \mathrm{~V}$, showing $1 / T$ dependence, indicating the origin of $1 / f$ noise to be from universal conductance fluctuations. (f) Schematic illustration of universal conductance fluctuations, which originates from quantum interference effect. 
large area TI samples are different [Figs. 3(a) and 3(b)]. The observed behavior of $1 / f$ noise on the charge carrier density has been predicted theoretically for Dirac Fermions for long range as well as Gaussian disorder, due to a crossover from pseudodiffusive to diffusive transition, which we believe is the scenario here [6]. In the pseudodiffusive regime, the transport in the channel occurs through quantum tunneling of evanescent modes. However, due to the presence of disorder, the system is driven into a diffusive metal phase, with the propagation occurring via plane waves. Although signatures of pseudodiffusive transport has been reported in graphene [8-22,58], there is no such clear signature in TIs. In the pseudodiffusive regime, $\left\langle\delta G^{2}\right\rangle$ enhances rapidly in magnitude compared to $\langle G\rangle$ with increasing $n$, while in the diffusive regime, $\left\langle\delta G^{2}\right\rangle$ is almost constant whereas $\langle G\rangle$ increases [6]. This leads to a nonmonotonic dependence of $1 / f$ noise magnitude on $n$, which is a generic property of crossover between these two regimes.

In order to understand the origin of $1 / f$ noise in mesoscopic TI-FETs, we have performed $V_{G}$ dependence of noise at various temperatures for both samples. We observe that the nonmonotonic behavior of $1 / f$ noise in sample BT1 is strongly dependent on $T$, and disappears for $T \sim 20 \mathrm{~K}$ [Fig. 3(c)], in contrast to graphene where the peak persists till room temperature [8]. The $V_{G}$ dependence of noise in sample BT2 shows a monotonic decrease with charge carrier density at all temperatures, and as demonstrated in Fig. 3(e), reduces as $\sim T^{-1}$, denoted by the red line for several $V_{G}$ 's. Such $T$ dependence of noise can be explained using the framework of universal conductance fluctuations (UCF), which arises due to quantum interference effects [36-38,59-61], and is schematically shown in Fig. 3(f). The charge carriers undergo multiple elastic scatterings from impurities, defects or boundaries, and follow trajectories which are a strong function of disorder configuration, Fermi energy, and magnetic field. Interference between these trajectories, which can involve backscattered carriers or interference between partial waves between two points having different paths leads to conductance fluctuations, whose noise spectra is $1 / f$ in nature [59]. For $T \rightarrow 0$, UCF magnitude $\left\langle\delta G^{2}\right\rangle^{\frac{1}{2}} \rightarrow e^{2} / h$, while at finite $T,\left\langle\delta G^{2}\right\rangle \simeq\left(\frac{e^{2}}{h}\right)^{2} \alpha\left(k_{F} \delta r\right) \frac{1}{k_{F} l_{m}} \frac{l_{y}}{l_{x}^{3}} n_{s}(T) l_{\phi}^{4}$, where $k_{F}, l_{m}, l_{x}$ and $l_{y}$ are the Fermi wave vector, mean free path, and sample dimensions in $x$ and $y$ directions, respectively [61-63]. $\alpha(x)$ represents the change of the phase of electron wave function due to scattering by a moving impurity at a distance $\delta r$, and $n_{s}(T)$ is the number of active scatterers. For electron-electron interaction mediated dephasing, $l_{\phi}^{2} \propto 1 / T$ and $n_{s}(T) \propto T$, we have $\left\langle\delta G^{2}\right\rangle \propto l_{\phi}^{4} n_{s}(T) \propto 1 / T$, as observed [61-64]. This leads us to conclude UCF as the dominant source of noise in thin, mesoscopic TIs at low $T$. This is contrary to what has been observed in large area epitaxially grown TIs, where the dominant source of noise is trapping-detrapping between the channel and the bulk defects [37]. The key difference between mesoscopic and epitaxial samples arises due to the difference in the ratio of channel dimensions and phase breaking length. Whereas in our samples, $l_{x} \sim l_{y} \sim l_{\phi}$, in case of the epitaxially grown samples, $l_{x}$, and $l_{y}$ are almost three orders of magnitude larger than $l_{\phi}$, which ensures that UCF is suppressed. For BT1, the overall noise magnitude reduces as $T$ is increased,
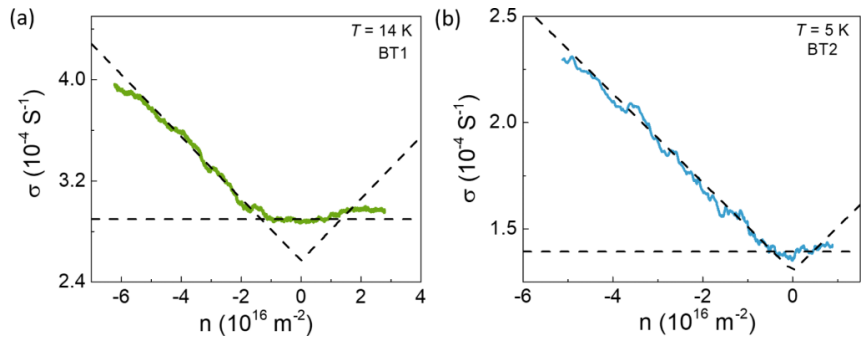

FIG. 4. Impurity density calculation. (a) Conductance $\left(\sigma=\frac{L}{R W}\right)$ vs calculated charge carrier density $\left(n_{\text {calc }}=\frac{C\left(V_{G}-V_{D}\right)}{e}\right)$ at $T=14 \mathrm{~K}$ for sample BT1. (b) $\sigma$ vs $n$ for sample BT2. The dashed lines in both (a), and (b) are fits to the data according to Eqs. (3) and (4).

although we do not observe any specific trend with $T$, such as that observed in BT2. This is not unexpected since the $T^{-1}$ dependence of noise magnitude arising from universal conductance fluctuations is strictly valid in the diffusive regime, which may not hold true in the pseudodiffusive transport scenario, and requires further theoretical understanding $[63,65]$.

\section{DISCUSSION}

To gain further insight into the nature of electrical transport in these mesoscopic samples, we have fitted $\sigma-n$ data [Fig. 4(a)], where $\sigma=\frac{L}{R W}$ and $n=\frac{C_{S}\left(V_{G}-V_{D}\right)}{e}$, within the framework of charge-impurity limited scattering of Dirac Fermions [66], where

$$
\sigma \sim E\left|\frac{n}{n_{\text {imp }}}\right|\left[e^{2} / h\right] \text { for } n>n^{*}
$$

and

$$
\sigma \sim E\left|\frac{n^{*}}{n_{\mathrm{imp}}}\right|\left[e^{2} / h\right] \text { for } n<n^{*},
$$

where $n_{\text {imp }}$ is the impurity density, $n^{*}$ is the residual charge carrier density in electron and hole puddles, and $E$ is a constant depending on the Wigner-Seitz radius $r_{s}$. The extracted value of density of Coulomb traps in BT1 is $n_{\mathrm{imp}}=$ $1.5 \times 10^{16} \mathrm{~m}^{-2}$, while for BT2, $n_{\text {imp }}=5 \times 10^{16} \mathrm{~m}^{2}$, which matches well with the theoretically predicted values [6]. The density of electron-hole puddles is $n^{*}=7 \times 10^{14} \mathrm{~m}^{-2}$ and $n^{*}=5 \times 10^{15} \mathrm{~m}^{-2}$ for samples BT1 and BT2, respectively. This difference in impurity density is reflected in the the qualitative nature of $V_{G}$ dependence of noise as seen in Figs. 3(a) and 3(b), thereby providing support to the observation of pseudodiffusive transport in device BT1. While the samples have been prepared following similar fabrication protocols, the difference in the $n_{\mathrm{imp}}$ can arise since the impurities (vacancies, antisite defects) are distributed randomly within the same single crystal [67].

\section{CONCLUSION}

In summary, we have measured time-dependent voltage fluctuations to extract the magnitude of $1 / f$ noise in mesoscopic topological insulators devices as a function of gate voltage and temperature. The temperature dependence 
implies that the noise originates from universal conductance fluctuations due to quantum interference effects. More importantly, the nonmonotonic dependence of noise on the number density in the lower disordered samples signifies a crossover from pseudodiffusive to diffusive transport regime, a phenomena unique to Dirac Fermions.
[1] M. Z. Hasan and C. L. Kane, Rev. Mod. Phys. 82, 3045 (2010).

[2] D. Hsieh, D. Qian, L. Wray, Y. Xia, Y. S. Hor, R. J. Cava, and M. Z. Hasan, Nature (London) 452, 970 (2008).

[3] P. Roushan, J. Seo, C. V. Parker, Y. S. Hor, D. Hsieh, D. Qian, A. Richardella, M. Z. Hasan, R. J. Cava, and A. Yazdani, Nature (London) 460, 1106 (2009).

[4] M. König, S. Wiedmann, C. Brüne, A. Roth, H. Buhmann, L. W. Molenkamp, X.-L. Qi, and S.-C. Zhang, Science 318, 766 (2007).

[5] H. Zhang, C.-X. Liu, X.-L. Qi, X. Dai, Z. Fang, and S.-C. Zhang, Nat. Phys. 5, 438 (2009).

[6] E. Rossi, J. H. Bardarson, M. S. Fuhrer, and S. Das Sarma, Phys. Rev. Lett. 109, 096801 (2012).

[7] H. Beidenkopf, P. Roushan, J. Seo, L. Gorman, I. Drozdov, Y. San Hor, R. J. Cava, and A. Yazdani, Nat. Phys. 7, 939 (2011).

[8] A. N. Pal, S. Ghatak, V. Kochat, E. Sneha, A. Sampathkumar, S. Raghavan, and A. Ghosh, ACS Nano 5, 2075 (2011).

[9] K. S. Novoselov, A. K. Geim, S. V. Morozov, D. Jiang, Y. Zhang, S. V. Dubonos, I. V. Grigorieva, and A. A. Firsov, Science 306, 666 (2004).

[10] K. Novoselov, D. Jiang, F. Schedin, T. Booth, V. Khotkevich, S. Morozov, and A. Geim, Proc. Natl. Acad. Sci. USA 102, 10451 (2005).

[11] A. H. Castro Neto, F. Guinea, N. M. R. Peres, K. S. Novoselov, and A. K. Geim, Rev. Mod. Phys. 81, 109 (2009).

[12] J. Tworzydło, B. Trauzettel, M. Titov, A. Rycerz, and C. W. J. Beenakker, Phys. Rev. Lett. 96, 246802 (2006).

[13] M. Katsnelson, Eur. Phys. J. B 51, 157 (2006).

[14] J. C. Cuevas and A. Levy Yeyati, Phys. Rev. B 74, 180501(R) (2006).

[15] A. R. Akhmerov and C. W. J. Beenakker, Phys. Rev. B 75, 045426 (2007).

[16] M. Titov and C. W. J. Beenakker, Phys. Rev. B 74, 041401(R) (2006).

[17] L. DiCarlo, J. R. Williams, Y. Zhang, D. T. McClure, and C. M. Marcus, Phys. Rev. Lett. 100, 156801 (2008).

[18] R. Danneau, F. Wu, M. F. Craciun, S. Russo, M. Y. Tomi, J. Salmilehto, A. F. Morpurgo, and P. J. Hakonen, Phys. Rev. Lett. 100, 196802 (2008).

[19] F. Miao, S. Wijeratne, Y. Zhang, U. Coskun, W. Bao, and C. Lau, Science 317, 1530 (2007).

[20] H. B. Heersche, P. Jarillo-Herrero, J. B. Oostinga, L. M. Vandersypen, and A. F. Morpurgo, Nature 446, 56 (2007).

[21] X. Du, I. Skachko, and E. Y. Andrei, Phys. Rev. B 77, 184507 (2008).

[22] P. Kumaravadivel and X. Du, Sci. Rep. 6, 24274 (2016).

[23] D. A. Abanin, K. S. Novoselov, U. Zeitler, P. A. Lee, A. K. Geim, and L. S. Levitov, Phys. Rev. Lett. 98, 196806 (2007).

[24] D. Abanin, S. Morozov, L. Ponomarenko, R. Gorbachev, A. Mayorov, M. Katsnelson, K. Watanabe, T. Taniguchi, K. Novoselov, L. Levitov et al., Science 332, 328 (2011).

[25] N. Peres, Rev. Mod. Phys. 82, 2673 (2010).
[26] Y.-W. Tan, Y. Zhang, K. Bolotin, Y. Zhao, S. Adam, E. H. Hwang, S. Das Sarma, H. L. Stormer, and P. Kim, Phys. Rev. Lett. 99, 246803 (2007).

[27] J. G. Checkelsky, L. Li, and N. P. Ong, Phy. Rev. B 79, 115434 (2009).

[28] Z. Jiang, Y. Zhang, H. L. Stormer, and P. Kim, Phys. Rev. Lett. 99, 106802 (2007).

[29] B. E. Feldman, J. Martin, and A. Yacoby, Nat. Phys. 5, 889 (2009).

[30] X. Du, I. Skachko, F. Duerr, A. Luican, and E. Y. Andrei, Nature (London) 462, 192 (2009).

[31] M. Weissman, Rev. Mod. Phys. 60, 537 (1988).

[32] M. B. Weissman, Annu. Rev. Mater. Sci. 26, 395 (1996).

[33] P. Dutta and P. Horn, Rev. Mod. Phys. 53, 497 (1981).

[34] P. Karnatak, T. Paul, S. Islam, and A. Ghosh, Adv. Phys. X 2, 428 (2017).

[35] S. Bhattacharyya, M. Banerjee, H. Nhalil, S. Islam, C. Dasgupta, S. Elizabeth, and A. Ghosh, ACS Nano 9, 12529 (2015).

[36] S. Bhattacharyya, A. Kandala, A. Richardella, S. Islam, N. Samarth, and A. Ghosh, Appl. Phys. Lett. 108, 082101 (2016).

[37] S. Islam, S. Bhattacharyya, A. Kandala, A. Richardella, N. Samarth, and A. Ghosh, Appl. Phys. Lett. 111, 062107 (2017).

[38] S. Islam, S. Bhattacharyya, H. Nhalil, S. Elizabeth, and A. Ghosh, Phys. Rev. B 97, 241412(R) (2018).

[39] S. Ghatak, A. N. Pal, and A. Ghosh, ACS Nano 5, 7707 (2011).

[40] S. Ghatak, S. Mukherjee, M. Jain, D. Sarma, and A. Ghosh, APL Mater. 2, 092515 (2014).

[41] S. Shamim, B. Weber, D. W. Thompson, M. Y. Simmons, and A. Ghosh, Nano Lett. 16, 5779 (2016).

[42] A. A. Taskin, Z. Ren, S. Sasaki, K. Segawa, and Y. Ando, Phys. Rev. Lett. 107, 016801 (2011).

[43] Z. Ren, A. A. Taskin, S. Sasaki, K. Segawa, and Y. Ando, Phys. Rev. B 84, 165311 (2011).

[44] C. R. Dean, A. F. Young, I. Meric, C. Lee, L. Wang, S. Sorgenfrei, K. Watanabe, T. Taniguchi, P. Kim, and K. L. Shepard, Nat. Nanotechnol. 5, 722 (2010).

[45] P. Karnatak, T. P. Sai, S. Goswami, S. Ghatak, S. Kaushal, and A. Ghosh, Nat. Commun. 7, 13703 (2016).

[46] S. Islam, S. Bhattacharyya, H. Nhalil, M. Banerjee, A. Richardella, A. Kandala, D. Sen, N. Samarth, S. Elizabeth, and A. Ghosh, Phys. Rev. B 99, 245407 (2019).

[47] S. Adam, E. H. Hwang, and S. Das Sarma, Phys. Rev. B 85, 235413 (2012).

[48] L. Zhang, M. Dolev, Q. I. Yang, R. H. Hammond, B. Zhou, A. Palevski, Y. Chen, and A. Kapitulnik, Phys. Rev. B 88, 121103(R) (2013).

[49] H.-T. He, G. Wang, T. Zhang, I.-K. Sou, G. K. L. Wong, J.-N. Wang, H.-Z. Lu, S.-Q. Shen, and F.-C. Zhang, Phys. Rev. Lett. 106, 166805 (2011).

[50] Y. S. Kim, M. Brahlek, N. Bansal, E. Edrey, G. A. Kapilevich, K. Iida, M. Tanimura, Y. Horibe, S.-W. Cheong, and S. Oh, Phys. Rev. B 84, 073109 (2011). 
[51] N. Bansal, Y. S. Kim, M. Brahlek, E. Edrey, and S. Oh, Phys. Rev. Lett. 109, 116804 (2012).

[52] S. Hikami, A. I. Larkin, and Y. Nagaoka, Prog. Theor. Phys. 63, 707 (1980).

[53] L. Bao, L. He, N. Meyer, X. Kou, P. Zhang, Z.-G. Chen, A. V. Fedorov, J. Zou, T. M. Riedemann, and T. A. Lograsso, Sci. Rep. 2, 726 (2012).

[54] J. H. Scofield, Rev. Sci. Instrum 58, 985 (1987).

[55] K. Hsieh, V. Kochat, X. Zhang, Y. Gong, C. S. Tiwary, P. M. Ajayan, and A. Ghosh, Nano Lett. 17, 5452 (2017).

[56] A. Ghosh, S. Kar, A. Bid, and A. K. Raychaudhuri, arXiv:condmat/0402130 (2004).

[57] R. Jayaraman and C. G. Sodini, IEEE Trans. Electron Devices 36, 1773 (1989).

[58] J. Dufouleur, L. Veyrat, B. Dassonneville, E. Xypakis, J. H. Bardarson, C. Nowka, S. Hampel, J. Schumann, B. Eichler, O. Schmidt et al., Sci. Rep. 7, 45276 (2017).
[59] P. A. Lee and A. D. Stone, Phys. Rev. Lett. 55, 1622 (1985).

[60] P. A. Lee, A. D. Stone, and H. Fukuyama, Phys. Rev. B 35, 1039 (1987).

[61] S. Feng, P. A. Lee, and A. D. Stone, Phys. Rev. Lett. 56, 1960 (1986).

[62] N. O. Birge, B. Golding, and W. H. Haemmerle, Phys. Rev. B 42, 2735 (1990).

[63] S. Shamim, S. Mahapatra, G. Scappucci, W. M. Klesse, M. Y. Simmons, and A. Ghosh, Sci. Rep. 7, 46670 (2017).

[64] B. L. Altshuler, A. Aronov, and D. Khmelnitsky, J. Phys. C: Solid State Phys. 15, 7367 (1982).

[65] C. Beenakker and H. van Houten, in Solid State Physics, Vol. 44 (Elsevier, Amsterdam, 1991), pp. 1-228.

[66] D. Kim, S. Cho, N. P. Butch, P. Syers, K. Kirshenbaum, S. Adam, J. Paglione, and M. S. Fuhrer, Nat. Phys. 8, 459 (2012).

[67] R. J. Cava, H. Ji, M. K. Fuccillo, Q. D. Gibson, and Y. S. Hor, J. Mater. Chem. C 1, 3176 (2013). 